\title{
Article
}

Mycosphere

Doi 10.5943/mycosphere/9/1/5

Copyright $\odot$ Guizhou Academy of Agricultural Sciences

\section{Orbiliaceae from Thailand}

\author{
Ekanayaka AH ${ }^{1,2}$, Hyde KD ${ }^{1,2}$, Jones EBG ${ }^{3}$, Zhao $Q^{1}$ \\ ${ }^{1}$ Key Laboratory for Plant Diversity and Biogeography of East Asia, Kunming Institute of Botany, Chinese Academy of \\ Sciences, Kunming 650201, Yunnan, China \\ ${ }^{2}$ Center of Excellence in Fungal Research, Mae Fah Luang University, Chiang Rai, 57100, Thailand \\ ${ }^{3}$ Department of Entomology and Plant Pathology, Faculty of Agriculture, Chiang Mai University, 50200, Thailand
}

Ekanayaka AH, Hyde KD, Jones EBG, Zhao Q 2018 - Orbiliaceae from Thailand. Mycosphere 9(1), 155-168, Doi 10.5943/mycosphere/9/1/5

\begin{abstract}
The family Orbiliaceae is characterized by small, yellowish, sessile to sub-stipitate apothecia, inoperculate asci and asymmetrical globose to fusoid ascospores. Morphological and phylogenetic studies were carried out on new collections of Orbiliaceae from Thailand and revealed Hyalorbilia erythrostigma, Hyalorbilia inflatula, Orbilia stipitata sp. nov., Orbilia leucostigma and Orbilia caudata. Our new species is confirmed to be divergent from other Orbiliaceae species based on morphological examination and molecular phylogenetic analyses of ITS and LSU sequence data. Descriptions and figures are provided for the taxa which are also compared with allied taxa.
\end{abstract}

Key words - apothecia - discomycetes - inoperculate - phylogeny - taxonomy

\section{Introduction}

The family Orbiliaceae was established by Nannfeldt (1932). Previously, this family has been treated as a member of Leotiomycetes (Korf 1973, Spooner 1987) and Eriksson et al. (2003) transferred this family into a new class Orbiliomycetes. The recent studies on this class include $\mathrm{Yu}$ et al. (2011), Guo et al. (2014), Qiao et al. (2015), Quijada et al. (2012, 2014, 2015, 2016), Quijada \& Baral (2017), Baral et al. (2017). A recent account of the family is provided by Ekanayaka et al. (2017). This family is wide spread, mostly saprobic on various plant substrates. Some species have the ability to trap invertebrates by means of adhesive devices or constricting rings ( $\mathrm{Li}$ et al. 2006).

The present study provides morphological descriptions of five Orbiliaceae species from Thailand, viz. Hyalorbilia erythrostigma, Hyalorbilia inflatula, Orbilia stipitata sp. nov., Orbilia leucostigma and Orbilia caudata. Phylogenetic analyses inferred from LSU and ITS sequence data support the molecular lineages for taxa of Orbilia, corresponding to morphological features.

\section{Materials \& Methods}

\section{Sample collection specimen examination and deposition}

Five specimens of Orbilia were collected from southern Thailand in 2014 to 2015. Macroscopic and microscopic characters of the specimens were recorded. A Motic SMZ-168 stereo microscope was used to observe structures of the apothecia. Thin hand sections of apothecia were made with a razor blade and mounted in water. A Nikon ECLIPSE 80i compound microscope was 
used to observe the microscopic characters. Photomicrography was carried out with a Canon 450D digital camera fitted to the microscope. Measurements of paraphyses, asci and ascospores were made from materials mounted in water and the mean values were used in the descriptions. Measurements were made with the Taro soft (R) Image Frame Work v. 0.9.7 program and images used for figures were processed with Adobe Photoshop CS6 software (Adobe Systems Inc.). The type specimens are deposited in the Mae Fah Luang University Herbarium (MFLU), Chiang Rai, Thailand and in the Herbarium of Cryptogams of Kunming Institute of Botany, Chinese Academy of Sciences (KUN-HKAS). Facesoffungi and Index Fungorum numbers were registered as described in Jayasiri et al. (2015) and Index Fungorum (2017).

\section{DNA extraction, PCR and sequencing}

Genomic DNA was extracted directly from the apothecia using a Plant DNA Rapid Extraction Kit (Bio Teke corporation, Beijing, China). Polymerase chain reactions (PCR) for this study were carried out for the internal transcribed spacer (ITS), using ITS4 and ITS5 (White et al. 1990) and LROR and LR5 (Vilgalys \& Hester 1990) for the nuclear ribosomal large subunit (LSU) primers. The PCR mixtures $(25 \mu \mathrm{L})$ contained $\mathrm{ddH}_{2} \mathrm{O}(11 \mu \mathrm{L})$, PCR Master Mix (QinKe Co., China) $(11 \mu \mathrm{L} ; 2 \times)$, DNA template $(1 \mu \mathrm{L})$, each primer $(1 \mu \mathrm{L} ; 10 \mu \mathrm{M})$. PCR amplification conditions for both gene regions were consisted an initial denaturation step of 5 min at $94{ }^{\circ} \mathrm{C}, 35$ cycles consisted of denaturation at $94{ }^{\circ} \mathrm{C}$ for 1 minute, annealing at $53{ }^{\circ} \mathrm{C}$ for 50 seconds and elongation at $72{ }^{\circ} \mathrm{C}$ for 3 minute and final extension step of 7 minutes at $72{ }^{\circ} \mathrm{C}$. The PCR products were viewed on $1 \%$ agarose electrophoresis gels, stained with ethidium bromide. PCR products were sent to a commercial sequencing provider, Qinke in Kunming, China.

\section{Sequence alignment and phylogenetic analysis}

Newly generated sequences in this study were subjected to a standard BLAST search of GenBank for rough identification. For the phylogenetic analysis, sequences belonging to ITS and LSU gene regions from representative Orbiliaceae species and the out-group taxon Tuber albidum Fr., were downloaded from GenBank (Table 1). The newly generated sequences are deposited in GenBank (Table 1). The consensus sequences for each gene were aligned using MAFFT v. 6.864b (http://mafft.cbrc.jp/alignment/server/index.html). The alignment was improved manually where necessary using Bioedit (Hall 2004). The model of evolution was estimated by using MrModeltest 2.2 (Nylander 2004). Maximum likelihood (ML) phylogenetic analyses were performed in the CIPRES web portal (Miller et al. 2010) using RAxML-HPC2 Workflow on XSEDE (8.2.9) tool. The bootstrap analysis for each ML tree was performed with 1000 thorough bootstrap replicates with the same parameter settings using combined alignment of ITS and LSU gene regions. The resultant trees were viewed with FigTree v.1.4.0 (http://tree.bio.ed.ac.uk/software/figtree/). Maximum parsimony analysis (MP) was performed with PAUP (Phylogenetic Analysis Using Parsimony) v. 4.0b10 (Swofford 2003) for the combined ITS and LSU gene regions. Ambiguously aligned regions were excluded from the analysis, gaps were treated as missing data. Trees were inferred using the heuristic search option with TBR branch swapping and 1000 random sequence additions. Branches of zero length were collapsed and all equally most parsimonious trees were saved. Descriptive tree statistics such as tree length [TL], consistency index $[\mathrm{CI}]$, retention index $[\mathrm{RI}]$, rescaled consistency index [RC], and homoplasy index [HI], were calculated.

Posterior probabilities in Bayesian inference (BI) (Rannala \& Yang 1996, Zhaxybayeva \& Gogarten 2002) were determined by Markov Chain Monte Carlo sampling (MCMC) in MrBayes v. 3.0b4 (Huelsenbeck \& Ronquist 2001). The GTR+ G substitution model selected by MrModel Test. Four simultaneous Markov chains were run for 25,000,000 generations and trees were sampled every 100th generation. The MCMC heated chain was set with a "temperature" value of 0.15 . The distribution of loglikelihood scores were examined to determine stationary phase for each search and to decide if extra runs were required to achieve convergence, using the program Tracer 1.5 (Rambaut \& Drummond 2009). All sampled topologies beneath the asymptote (20\%) were 
discarded as part of a burn-in procedure, while the remaining trees $(20,000,000)$ were used for calculating posterior probabilities in the majority rule consensus tree.

The resultant trees were viewed with FigTree v.1.4.0 (http://tree.bio.ed.ac.uk/software/figtree/). Bayesian posterior probabilities (BPP) equal or greater than 0.9 are given as the first set of numbers above the nodes, Maximum likelihood bootstrap values (MLBP) equal or greater than $70 \%$ are given as the second set of numbers above the nodes and maximum-parsimony bootstrap values (MPBP) equal or greater than $70 \%$ are given as the third set of numbers near the nodes (Fig. 1).

Table 1 Taxa used in the two gene phylogenetic analyses and ITS and LSU GenBank accession numbers (Newly generated sequences are in bold).

\begin{tabular}{|c|c|c|}
\hline Species name & Strain number & GenBank number \\
\hline Amphosoma atroolivacea & G.M. 2010-09-03 & KT380069 \\
\hline Amphosoma resinicola & H.B. $6992 \mathrm{a}$ & KT222389 \\
\hline Arthobotrys superba & CBS 10952 & KT215210 \\
\hline Arthrobotrys flagrans & CBS 58391 & AY261132/AF106520 \\
\hline Arthrobotrys oligospora & ATCC 96709 & EF445989 \\
\hline Arthrobotrys scaphoides & CBS 22652 & NR_145361 \\
\hline Brachyphoris brevistipitata & CBS 113946 & KT215221 \\
\hline Brachyphoris lignatilis & YMFT 1.00596 & KT215222 \\
\hline Dactylella cylindrospora & CBS 32570 & NR_145394 \\
\hline Dactylella intermedia & CBS109506 & DQ494359 \\
\hline Dactylella zhongdianensis & H.B. 9641 & KT222436 \\
\hline Dactylellina leptospora & CBS 100579 & KT215199 \\
\hline Dactylellina mammillata & CBS229.54 & AY902802/AY902794 \\
\hline Dactylellina parvicollis & CBS 31794 & KT215206 \\
\hline Dactylellina pauca & CBS 64280 & KT215205 \\
\hline Drechslerella bembicodes & HB $7165 b$ & KT215295 \\
\hline Drechslerella brochopaga & ATCC 96710 & EF445987 \\
\hline Drechslerella dactyloides & CNU091026 & GU130301/GU130300 \\
\hline Drechslerella doedycoides & ATCC 96778 & EF445992 \\
\hline Gamsylella arcuata & CBS 17489 & KT215197 \\
\hline Gamsylella gephyropaga & ATCC 96677 & EF445990 \\
\hline Gamsylella lobata & CBS 329.94 & AF106524 \\
\hline Gamsylella phymatopaga & CBS 56895 & KT215203 \\
\hline Helicoon sessile & A 327 & KY659207 \\
\hline Hyalorbilia cf. inflatula & MFLU 15-0167/ HD007 & MG599270/MG599273 \\
\hline Hyalorbilia latispora & H.B. $9902 \mathrm{c}$ & KT222386 \\
\hline Hyalorbilia polypori & HB 7557 & KT215223 \\
\hline Hyalorbilia ulicicola & HMAS 139526 & DQ656691/DQ656646 \\
\hline Lecophagus longisporus & CBS 84591 & KT215220 \\
\hline Lilapila oculispora & H.B. 9631a & KT222383 \\
\hline Lilapila oculispora & G.M. 2011-09-19 & KT222384 \\
\hline Orbilia aethiopica & HB 9246a & KT222424 \\
\hline Orbilia alba & H.B. $9645 \mathrm{a}$ & KT222438 \\
\hline Orbilia albidorosea & HB 6615a & KT215254 \\
\hline
\end{tabular}


Table 1 Continued.

\begin{tabular}{|c|c|c|}
\hline Species name & Strain number & GenBank number \\
\hline Orbilia albovinosa & HB 7231f & KT215255 \\
\hline Orbilia anigozanthi & HB 8831 & KT222374 \\
\hline Orbilia aprilis & HB 6801 & KT215268 \\
\hline Orbilia aristata & HB 6713 & KT596782 \\
\hline Orbilia asomatica & TFCMic 21258 & KT222399 \\
\hline Orbilia aurantiorubra & HB 6815a & KF741595 \\
\hline Orbilia auricolor & HB 6664 & KT215294 \\
\hline Orbilia australiensis & HB 7208b & KT215262 \\
\hline Orbilia beltraniae & TFC Mic. 24363 & KT222405 \\
\hline Orbilia beltraniae & TFC Mic. 23890 & KT222406 \\
\hline Orbilia bomiensis & - & DQ656686/DQ656629 \\
\hline Orbilia brachychiti & HB 7578a & KT215257 \\
\hline Orbilia canadensis & HB 6826 & KT215277 \\
\hline Orbilia cardui & H.B. 9891 & KT222402 \\
\hline Orbilia carminorosea & HB 8777a & KT222423 \\
\hline Orbilia carpoboloides & HB 6639c & KT215248 \\
\hline Orbilia caulicola & G.M. 2013-07-30 & KT380067/KT380063 \\
\hline Orbilia caudata & MFLU 16-0580/ HD018 & MG599271/MG599274 \\
\hline Orbilia clavuliformis & HB 6714 & KT215271 \\
\hline Orbilia comma & HB 6639b & KT215258 \\
\hline Orbilia corculispora & НВ 6279 & KT215273 \\
\hline Orbilia cotoneastri & CBS 116281 & KT215288 \\
\hline Orbilia crenatomarginata & HB 9265 & KM248772 \\
\hline Orbilia cryptogena & HB 7397a & KT215272 \\
\hline Orbilia cucumispora & HB 6762 & KT215231 \\
\hline Orbilia denticulata & HB 6725 & KT215256 \\
\hline Orbilia digitalina & HB 7566a & KT596781 \\
\hline Orbilia dryadum & HB 6876a & KT215281 \\
\hline Orbilia ebuli & HB 9550 & KT222425 \\
\hline Orbilia epipora & HB 9039 & KT222431 \\
\hline Orbilia eucalypti & HB 6662 & KT215285 \\
\hline Orbilia eиonymi & H.B. 9152 & KT222444 \\
\hline Orbilia farnesianae & HB $8997 \mathrm{~h}$ & KT222421 \\
\hline Orbilia flavida & HB 6716 & KT215228 \\
\hline Orbilia flavidorosella & G.M. 2012-9-18 & KT222391 \\
\hline Orbilia fusiformis & YMF1.01848 & EF026115/EF026114 \\
\hline Orbilia gambelii & HB 6466 & KT215249 \\
\hline Orbilia hesperidea & HB 6694 & KT215263 \\
\hline Orbilia inaequalis & HB 6963a & KT215283 \\
\hline Orbilia lentiformis & HB $6569 c$ & KT215225 \\
\hline Orbilia leucostigma & HB 6810 & KT215282 \\
\hline Orbilia liliputiana & HB 6905 & KT215284 \\
\hline Orbilia luteorubella & YMF1.01843 & DQ480728/DQ480727 \\
\hline
\end{tabular}


Table 1 Continued.

\begin{tabular}{|c|c|c|}
\hline Species name & Strain number & GenBank number \\
\hline Orbilia mammillata & H.B. $7165 c$ & KT215290 \\
\hline Orbilia microserpens & HB 6519a & KT215251 \\
\hline Orbilia microsoma & HB 6738a & KT215278 \\
\hline Orbilia multimaeandrina & HB $6737 a$ & KT215243 \\
\hline Orbilia multimicrosoma & HB 9582a & KT380094 \\
\hline Orbilia multiurosperma & HB 6493a & KT215245 \\
\hline Orbilia myriofusiclava & HB 7237a & KT215274 \\
\hline Orbilia myriosphaera & HB 6679a & KT215233 \\
\hline Orbilia nemaspora & sp.4201 & DQ656700/DQ656630 \\
\hline Orbilia octoserpentina & HB $6609 b$ & KT215237 \\
\hline Orbilia oudemansii & HB 6972a & KT215292 \\
\hline Orbilia ovoidea & HB 6489a & KT215275 \\
\hline Orbilia paracylindrospora & HB 9484 & KT222430 \\
\hline Orbilia patellarioides & HB 6490a & KT215224 \\
\hline Orbilia phragmotricha & HB 7535a & KT215259 \\
\hline Orbilia pilifera & HB 8362a & KT222364 \\
\hline Orbilia pleioaustraliensis & HB $6277 \mathrm{e}$ & KT215261 \\
\hline Orbilia plesteuonymi & HB 6494a & KT215234 \\
\hline Orbilia pluristomachia & HB $6617 \mathrm{a}$ & KT215232 \\
\hline Orbilia polyspora & HB $7243 b$ & KT215276 \\
\hline Orbilia quaestiformis & HB 7121 & KT215246 \\
\hline Orbilia quercus & HMAS 88783 & DQ656669/AY804213 \\
\hline Orbilia rectispora & HB 7142 & KT215289 \\
\hline Orbilia rosea & HB 6756a & KM199779 \\
\hline Orbilia rubrovacuolata & HB 6598a & KT215291 \\
\hline Orbilia sarraziniana & HB 7235 & KM199780 \\
\hline Orbilia serpentina & HB 6609d & KT215238 \\
\hline Orbilia spermoides & HB 7517 & KT380068 \\
\hline Orbilia sphaerospora & HB 9129 & KT222429 \\
\hline Orbilia stipitata & MFLU 15-0229/ HD011 & MG599272/MG599275 \\
\hline Orbilia subaristata & H.B. $6685 \mathrm{a}$ & KT215270 \\
\hline Orbilia subocellata & H.B. 6474 & KT215227 \\
\hline Orbilia subvinosa & HB $6748 f$ & KT215265 \\
\hline Orbilia subvitalbae & HB 6504a & KT215250 \\
\hline Orbilia vinosa & AFTOL-ID 905 & DQ470952/DQ491511 \\
\hline Orbilia xanthoguttulata & Oxa79 & KF768637 \\
\hline Orbilia xinjiangensis & HB 9646 & KT222435 \\
\hline Tridentaria implicans & CBS 32056 & KT215279 \\
\hline Tridentaria subuliphora & CBS 70093 & KT215280 \\
\hline Tuber albidum & CBS 27272 & KT215193 \\
\hline Vermispora fusarina & CBS 38284 & HQ110699/DQ494377 \\
\hline
\end{tabular}




\section{Results}

\section{Phylogenetic analyses}

A phylogenetic tree for Orbilia species was generated using the combined ITS and LSU dataset and comprised 1632 characters including alignment gaps for 117 ingroup and one outgroup taxon. Of the 1632 characters, 563 were constant, 229 were variable and parsimony uninformative. Maximum parsimony analysis of the remaining 840 parsimony-informative characters resulted in most parsimonious tree $(\mathrm{TL}=10400 ; \mathrm{CI}=0.223, \mathrm{RI}=0.501, \mathrm{RC}=0.111, \mathrm{HI}=0.777)$. The final likelihood value of best scoring tree was -43441.239297 . The matrix had 1217 distinct alignment patterns, with $29.52 \%$ of undetermined characters or gaps. Estimated base frequencies were as follows; $\mathrm{A}=0.262, \mathrm{C}=0.227, \mathrm{G}=0.261, \mathrm{~T}=0.251$; substitution rates $\mathrm{AC}=1.347511, \mathrm{AG}=$ $1.974455, \mathrm{AT}=1.253581, \mathrm{CG}=0.889264, \mathrm{CT}=4.161195, \mathrm{GT}=1.000000$; gamma distribution shape parameter $\alpha=0.384574$. The trees from MP, ML and BI analyses showed the same topologies (Fig. 1).

\section{Taxonomy}

Orbilia stipitata Ekanayaka \& K.D. Hyde, sp. nov.

Fig. 2

Index Fungorum number: IF554114; Facesoffungi number: FoF 03954

Etymology - The specific epithet stipitata refers to the ascospore shape.

Holotype - MFLU 15-0229

Description - Saprobic on wood. Sexual morph: Apothecia 0.8-1.2 mm diam., scattered, yellow to cream, disc flat, superficial, margin smooth, discoid with a wide attachment subsessile to shortly stipitate. Ectal excipulum $18-23 \mu \mathrm{m}$ wide $(\bar{x}=21 \mu \mathrm{m}, \mathrm{n}=10)$ at upper flanks, composed of thin-walled textura globulosa. Medullary excipulum $30-45 \mu \mathrm{m}$ wide $(\bar{x}=36 \mu \mathrm{m}, \mathrm{n}=10)$ with few cell layers comprising with hyaline, gelatinized cells of textura angularis, sharply delimited from ectal excipulum. Hymenium hyaline. Paraphyses medium to strongly clavate, septate, unbranched, swollen at the apices, terminal cell $1-1.5 \mu \mathrm{m}$ width $(\bar{x}=1.3 \mu \mathrm{m}, \mathrm{n}=20)$. Asci $28-35 \times 4-5 \mu \mathrm{m}(\bar{x}=$ $31.8 \times 4.6 \mu \mathrm{m}, \mathrm{n}=30), 8$-spored, with thin apex; cylindric-clavate, with a short- to long-stalked bifurcate base. Ascospores 8-11 $\times 0.8-1.3 \mu \mathrm{m}(\bar{x}=9.8 \times 1.1 \mu \mathrm{m}, \mathrm{n}=40), 2-3$-seriate, cylindricsubcylindric, immature spores are straight and mature spores are strongly curved, apex obtuse, 1-2 guttules present at both poles. Asexual morph: Unknown.

Material examined - THAILAND, Kun Korn waterfall, Chiang Rai Province, on unidentified wood, 20 January 2015, A.H. Ekanayaka, HD0011 (MFLU 15-0229, holotype).

Notes: - Orbilia stipitata is morphologically similar to O. vermiformis and $O$. arcospora (Yu et al. 2007, Su et al. 2011). However, it differs by having larger (O. vermiformis, 39.3-62 $\times$ 3.8-5 $\mu \mathrm{m} ;$ O. arcospora, 42-53 $\times 3.4-4.4 \mu \mathrm{m}$ ) asci (Yu et al. 2007, Su et al. 2011). Orbilia stipitata is phylogenetically close to O. nemaspora 4201 (Baral et al. 2017), but they differ in 15 base pairs in the ITS and LSU regions.

Hyalorbilia cf. inflatula (P. Karst.) Baral \& G. Marson

Fig. 3

Facesoffungi number: FoF03955

Description - Saprobic on wood. Sexual morph: Apothecia 0.4-2 mm diam., gregarious, brownish yellow, discoid to deeply cupulate when mature, superficial, margin smooth, sessile with wide attachment. Ectal excipulum 10-15 $\mu \mathrm{m}$ wide at lover flanks, composed of large, thin-walled, yellowish cells of textura angularis-prismatica. Medullary excipulum 38-45 $\mu \mathrm{m}$ wide at lover flanks, composed of large, thin-walled, hyaline, gelatinized cells of textura angularis-prismatica, not sharply delimited from ectal excipulum. Hymenium hyaline, asci and paraphyses glued together and made a thick layer. Paraphyses uninflated, slightly septate at the base, unbranched, terminal cell $2-3 \mu \mathrm{m}$ wide $(\bar{x}=1.8 \mu \mathrm{m}, \mathrm{n}=20)$. Asci $25-30 \times 3-4 \mu \mathrm{m}(\bar{x}=25.9 \times 3.6 \mu \mathrm{m}, \mathrm{n}=30)$, 8 spored, biseriate, 


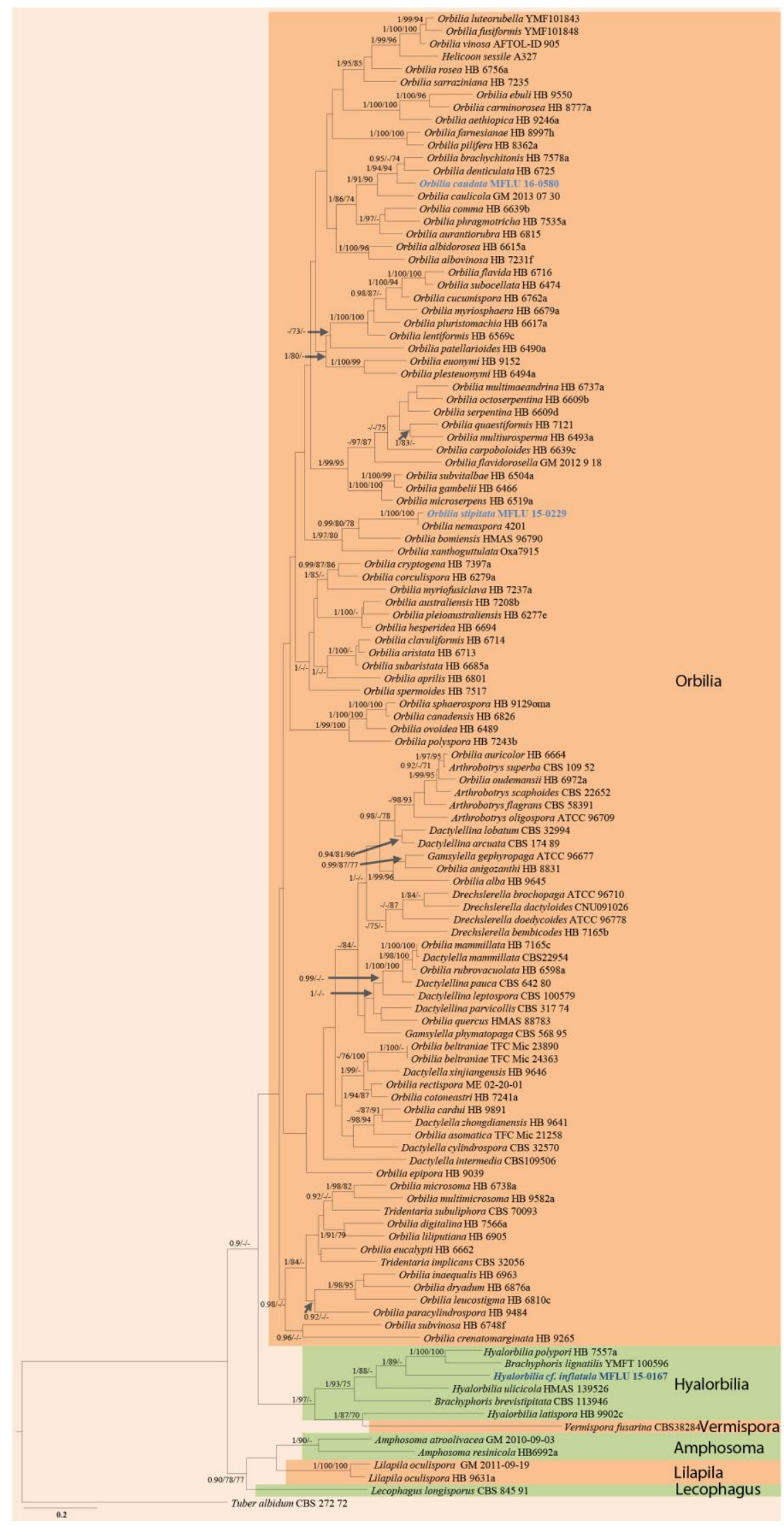

Figure 1 - Phylogram generated from maximum likelihood analysis of sequences of Orbiliaceae based on ITS and LSU sequence data. BPP $\geq 0.90$ are given as the first set of numbers, MLBP $\geq 70 \%$ are given as the second set of numbers and MPBP $\geq 70 \%$ are given as the third set of numbers, near the nodes. Strain/culture numbers are given after the taxon names. The newly generated sequences are in blue bold. The tree was rooted with Tuber albidum (CBS 272-72). 


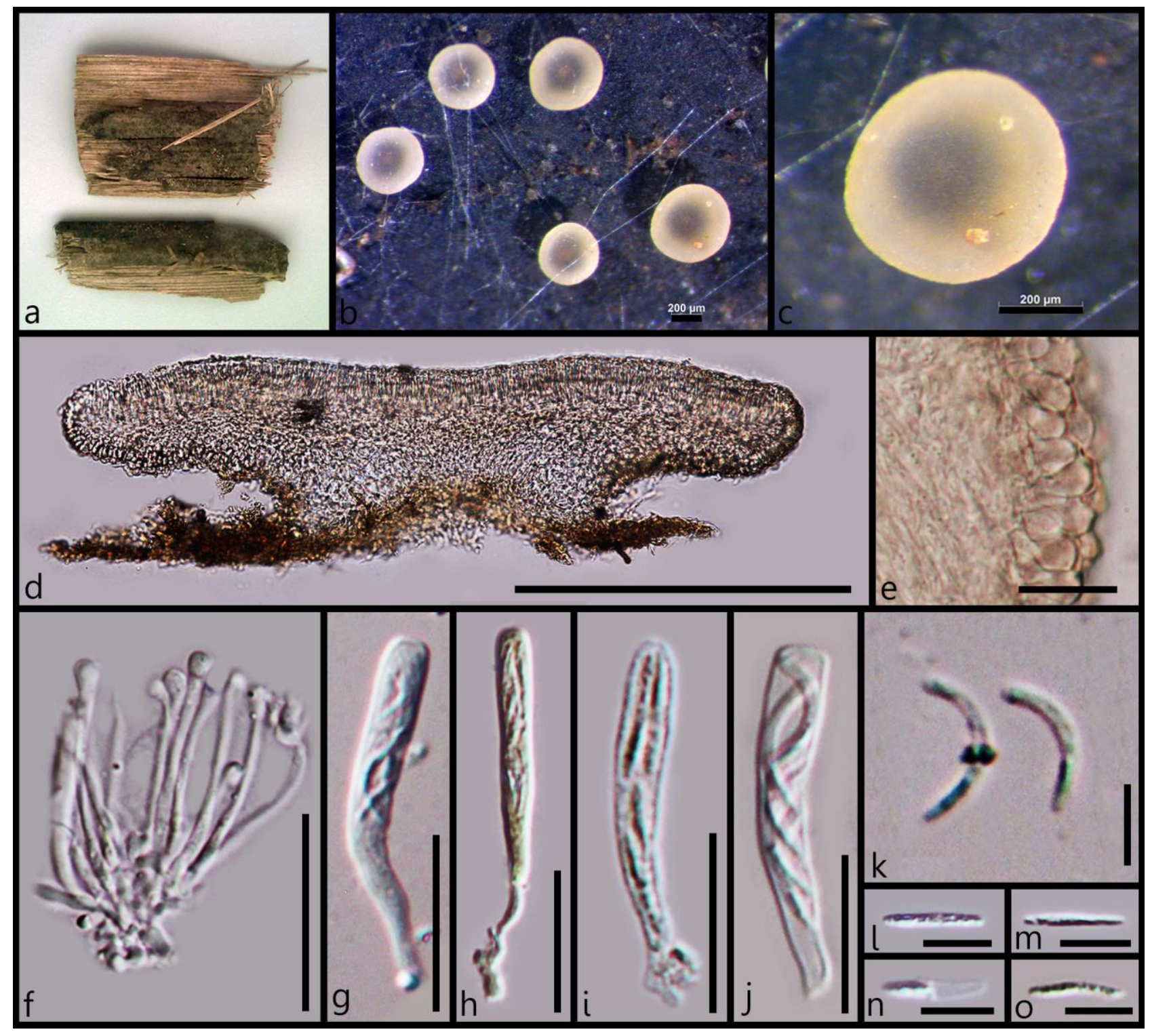

Figure 2 - Morphology of Orbilia stipitata (Holotype MFLU 15-0229) a Substrate. b,c Fresh apothecia. d Median section of an apothecia. e Excipular cells at upper flanks. f Paraphyses. g, h, i, j Asci, k-o: Ascospores. Scale bars: $\mathrm{b}, \mathrm{c}=200 \mu \mathrm{m}, \mathrm{d}=300 \mu \mathrm{m}, \mathrm{e}=15 \mu \mathrm{m}, \mathrm{f}=10 \mu \mathrm{m}, \mathrm{g}-\mathrm{j}=15 \mu \mathrm{m}$, $\mathrm{k}-\mathrm{o}=5 \mu \mathrm{m}$.

cylindric-clavate, 2-3-seriate, short pedicellate with croziers. Ascospores $4.5-5.5 \times 0.5-1.3 \mu \mathrm{m}(\bar{x}$ $=5.2 \times 1.1 \mu \mathrm{m}, \mathrm{n}=40$ ), cylindrical, strongly to slightly curved, both ends rounded to obtuse, eguttulate or 1-2 guttules present. Asexual morph: Undetermined.

Material examined - THAILAND, Mae Fah Luang University, Chiang Rai Province, on unidentified wood, 15 January 2015, A.H. Ekanayaka, HD007 (MFLU 15-0167).

Notes - Sequence data from type specimen of $H$. inflatula is not available. However, the description for $H$. inflatula in Quijada et al. (2015) is identical with our isolate. Therefore, we place our collection under $H$. inflatula. For details about differences of $H$. inflatula with morphological related species see Quijada et al. (2015).

Orbilia caudata Starbäck Bih. Kongl. Svenska Vetensk.-Akad. Handl., Afd. 3, 25(1): 8, 1899.

Facesoffungi number: FoF03956

Fig. 4

Description - Saprobic on wood. Sexual morph: Apothecia 0.2-0.7 mm diam., superficial, scattered to subgregarious, yellow to orange, margin smooth, disc flat, superficial, sessile with wide 
attachment. Ectal excipulum 12-16 $\mu \mathrm{m}$ wide at lover flanks, composed of large, thin-walled, yellowish cells of textura angularis-globulosa. Medullary excipulum 28-35 $\mu \mathrm{m}$ wide at lover flanks, composed of large, thin-walled, hyaline, gelatinized cells of textura angularis-intricata, not sharply delimited from ectal excipulum. Hymenium hyaline. Paraphyses $1.5-2.0 \mu \mathrm{m}$ wide $(\bar{x}=$ $1.8 \mu \mathrm{m}, \mathrm{n}=20)$ at the middle, medium clavate, numerous, filamentous, septate, branched at the base, slightly swollen at the apices, terminal cell $2-3 \mu \mathrm{m}$ wide $(\bar{x}=2.7 \mu \mathrm{m}, \mathrm{n}=20)$. Asci $30-40 \times 4.0$ $5.5 \mu \mathrm{m}(\bar{x}=34.7 \times 5.1 \mu \mathrm{m}, \mathrm{n}=30) 8$-spored, 1-2-seriate, cylindrical, apices truncate with a longstalked bifurcate base. Ascospores $7-9 \times 1.4-2 \mu \mathrm{m}(\bar{x}=8.1 \times 1.6 \mu \mathrm{m}, \mathrm{n}=40)$, asymmetrical, fusoid to ellipsoid, apex obtuse, tapered, sometimes form a inflated tail, straight to slightly-medium curved, base obtuse, hyaline, aseptate, smooth-walled, guttulate. Asexual morph: Undetermined.

Material examined - THAILAND, Mae Fah Luang University, Chiang Rai Province, on unidentified wood, 18 March 2015, A.H. Ekanayaka, HD018 (MFLU 16-0580).

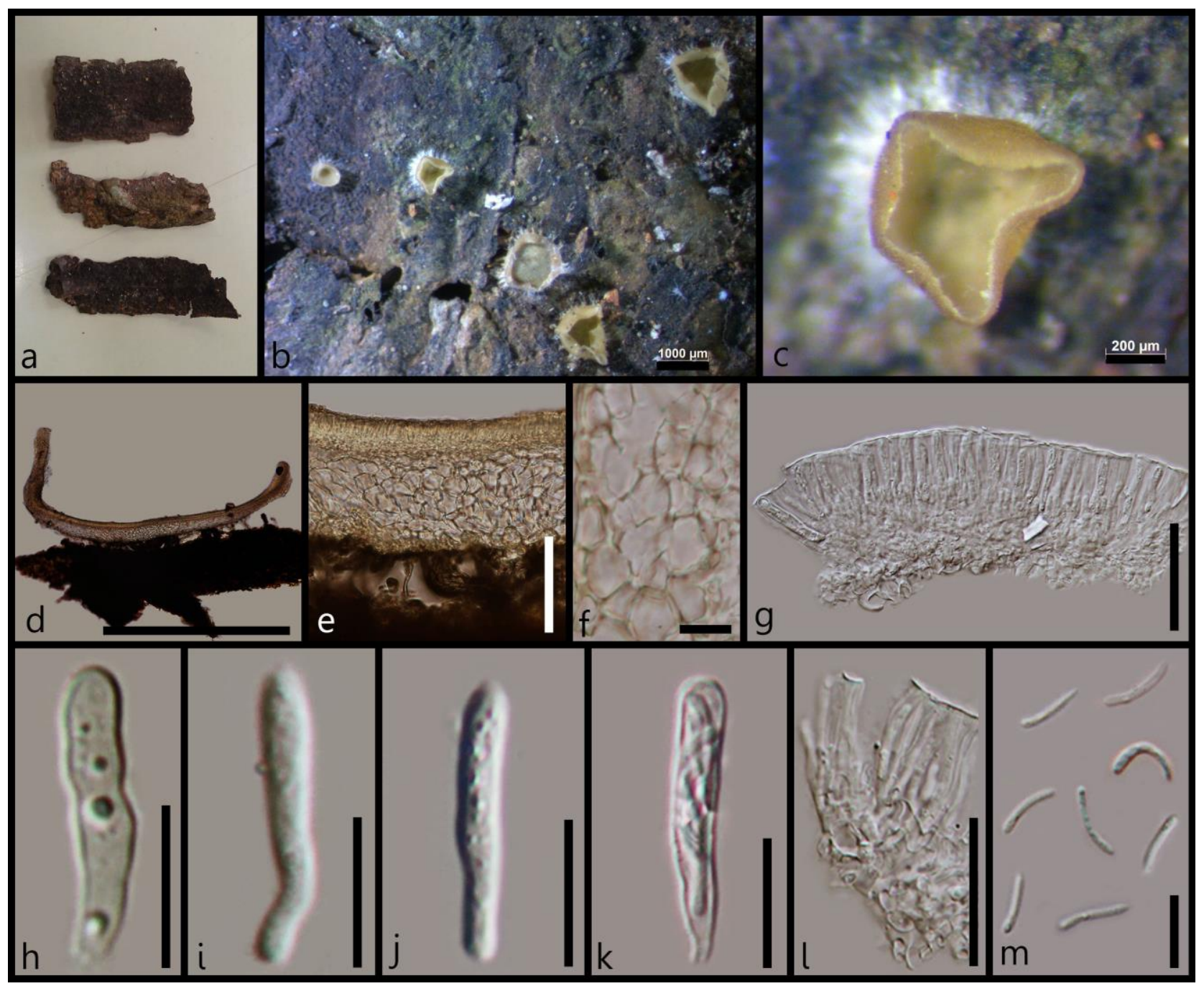

Figure 3 - Morphology of Hyalorbilia inflatula (MFLU 15-0167) a Substrate. b, c Rehydrated apothecia. d Median section of an apothecia. e, f Excipular cells at lover flanks. $g$ Hymenium layer. h, i, j, k Asci. 1 Paraphyses. m Ascospores. Scale bars: $b=1000 \mu \mathrm{m}, \mathrm{c}=200 \mu \mathrm{m}, \mathrm{d}=1000 \mu \mathrm{m}$, $\mathrm{e}=75 \mu \mathrm{m}, \mathrm{f}=10 \mu \mathrm{m}, \mathrm{g}=40 \mu \mathrm{m}, \mathrm{h}-\mathrm{k}=15 \mu \mathrm{m}, \mathrm{l}=25 \mu \mathrm{m}, \mathrm{m}=05 \mu \mathrm{m}$.

Notes - Orbilia pisciformis is similar to $O$. caudata mainly in ascospore shape, but differs in having straight ascospores with subacute apices (Quijada et al. 2012). Orbilia caudata is morphologically and phylogenetically close to O. comma. However, O. comma differs from $O$. caudata by having shorter ascospores $(5.8-7.6 \times 1.4-1.7 \mu \mathrm{m})$ and longer asci $(47-65 \times 4-4.8 \mu \mathrm{m})$ (Zhang et al. 2009, Quijada et al. 2012). 


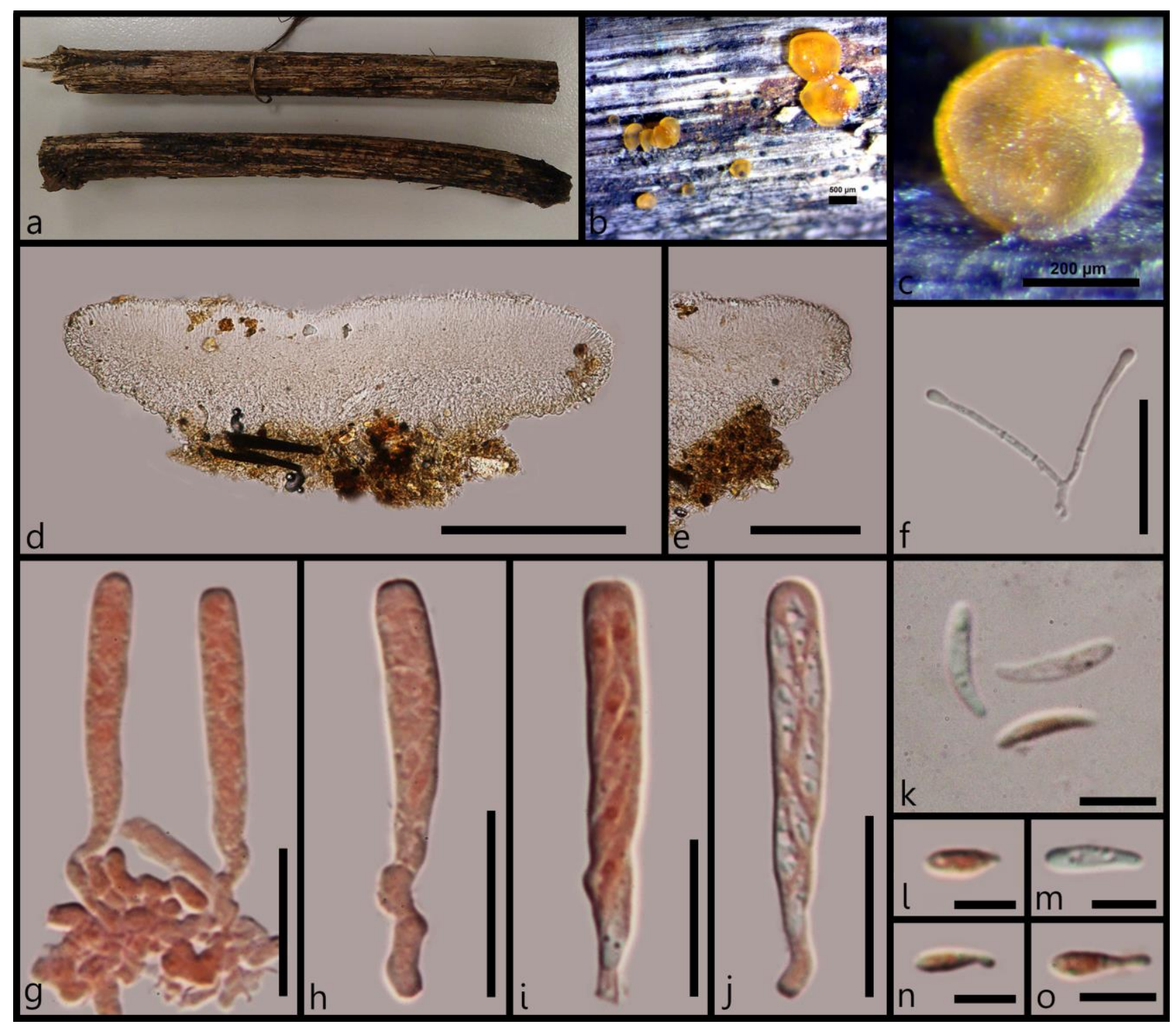

Figure 4 - Morphology of Orbilia caudata (MFLU 16-0580) a Substrate. b,c Fresh apothecia. d Median section of an apothecia. e Excipular cells at upper flanks. f Paraphyses. g-j Asci. k-o Ascospores. Scale bars: $\mathrm{b}=500 \mu \mathrm{m}, \mathrm{c}, \mathrm{d}=200 \mu \mathrm{m}, \mathrm{e}=100 \mu \mathrm{m}, \mathrm{f}=20 \mu \mathrm{m}, \mathrm{g}-\mathrm{j}=15 \mu \mathrm{m}, \mathrm{k}-\mathrm{o}=5 \mu \mathrm{m}$.

Hyalorbilia erythrostigma (W. Phillips) Baral \& G. Marson 2001

Fig. 5

Facesoffungi number: FoF03957

Description - Fungicolous on Annulohypoxylon sp. Sexual morph: Apothecia 220-250 × 380-430 $\mu \mathrm{m}$ (height $\times$ width) superficial, scattered to subgregarious, bright yellow, short stipitate, disc slightly concave, superficial, margin smooth. Ectal excipulum 9-14 $\mu \mathrm{m}$ wide at margins and upper flanks, composed of large, thin-walled, hyaline cells of textura angularis-globulosa. Medullary excipulum 18-25 $\mu \mathrm{m}$ wide at lover flanks, composed of large, thin-walled, hyaline, gelatinized cells of textura angularis-intricata, not sharply delimited from ectal excipulum. Hymenium hyaline, asci and paraphyses glued together and made a thick gelatinous layer. Paraphyses slightly to medium clavate, septate, terminal cell $1.8-2.5 \mu \mathrm{m}$ width $(\bar{x}=2.2 \mu \mathrm{m}$, $\mathrm{n}=20)$, unbranched. Asci 30-33 $\times 3-3.5 \mu \mathrm{m}(\bar{x}=31.4 \times 3.3 \mu \mathrm{m}, \mathrm{n}=30)$ 8-spored, cylindric clavate, short pedicellate, arising from croziers, truncate apex uniseriate. Ascospore 1.6-2.2 $\times 1.7-2.3 \mu \mathrm{m}$ $(\bar{x}=2.0 \times 2.1 \mu \mathrm{m}, \mathrm{n}=40)$, globose, subglobose to ellipsoid, hyaline, aseptate, 1-2 guttules present, smooth-walled. Asexual morph: Undetermined.

Material examined - THAILAND, Mae Fah Luang University, Chiang Rai Province, on Annulohypoxylon sp., 20 April 2015, A.H. Ekanayaka, HD028 (MFLU 16-0589). 
Notes - Hyalorbilia brevistipitata is morphologically similar to H. erythrostigma. However, $H$. brevistipitata differs from $H$. erythrostigma by having flat apothecia, and shorter stipe, smaller asci (18-25 $\times 3-3.5 \mu \mathrm{m})$ and smaller ascospores $(1.5-1.8 \times 1.5-1.8 \mu \mathrm{m})($ Liu et al. 2005). We were not able to obtain molecular data for this collection.

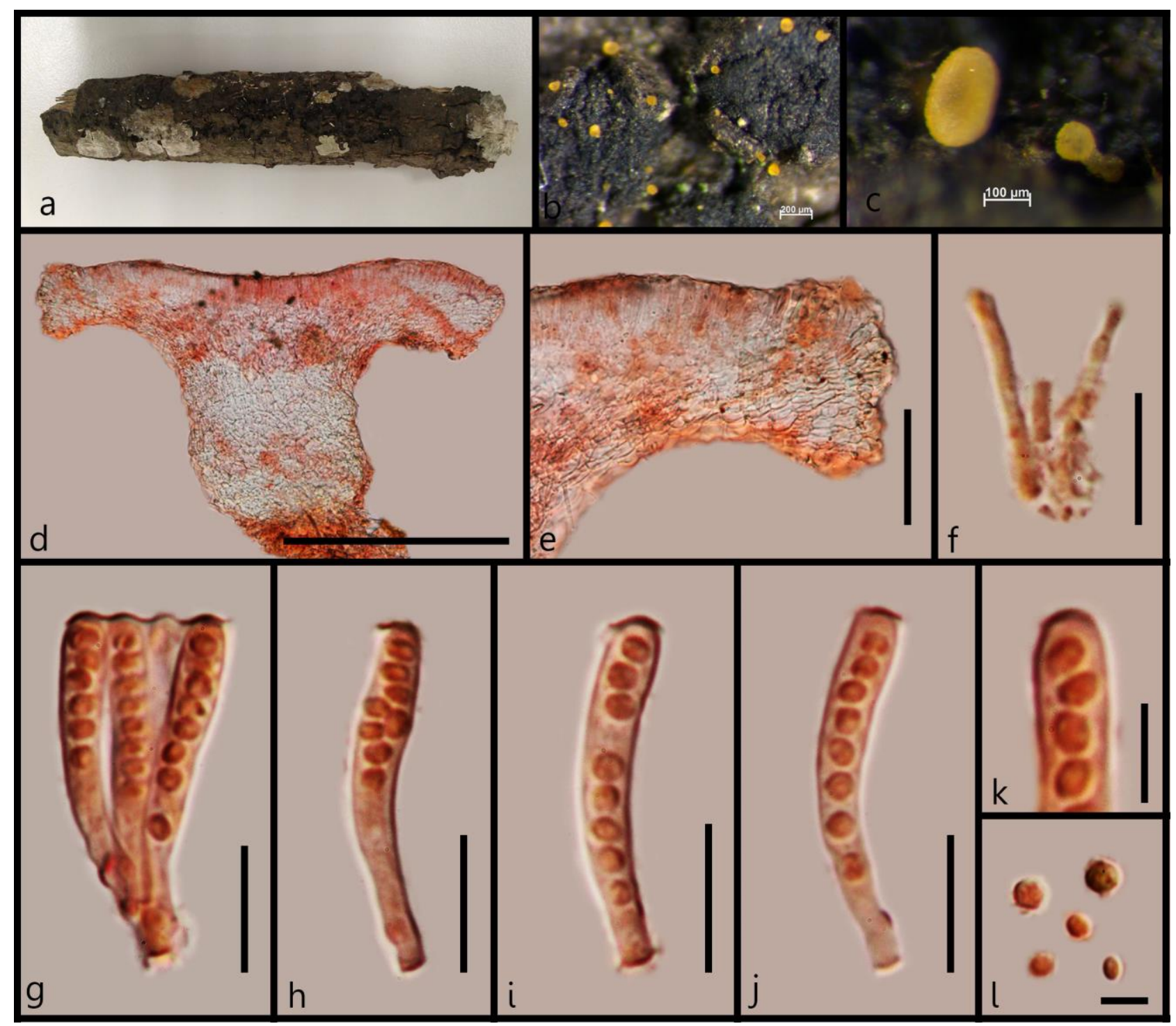

Figure 5 - Morphology of Hyalorbilia erythrostigma (MFLU 16-0589) a Substrate. b,c. Fresh apothecia. d Median section of an apothecia. e Excipular cells at upper flanks. f Paraphyses. g, h, i, j Asci. k-o Ascospores. (Structures are mounted in Congo red reagent). Scale bars: $b, d=200 \mu \mathrm{m}, \mathrm{c}$ $=100 \mu \mathrm{m}, \mathrm{e}=40 \mu \mathrm{m}, \mathrm{f}-\mathrm{j}=10 \mu \mathrm{m}, \mathrm{k}-\mathrm{l}=5 \mu \mathrm{m}$.

Orbilia leucostigma (Fr.) Fr., Summa veg. Scand., Sectio Post. (Stockholm): 357 (1849)

Fig. 6

Facesoffungi number: FoF03958

Description - Saprobic on wood. Sexual morph: Apothecia 350-400 $\mu \mathrm{m}$, superficial, sessile, scattered, yellow, sessile, disc flat or slightly concave, margin smooth. Receptacle turbinate yellow when fresh, yellowish orange when dry. Excipulum 15-20 $\mu \mathrm{m}$ wide $(\bar{x}=18 \mu \mathrm{m}, \mathrm{n}=10)$ Ectal excipulum composed of large, thin-walled cells of textura angularis. Medullary excipulum 25-30 $\mu \mathrm{m}$ wide $(\bar{x}=28 \mu \mathrm{m}, \mathrm{n}=10)$ hyaline cells of textura angularis to intricata. Hymenium hyaline. Paraphyses $1-2 \mu \mathrm{m}$ wide at the middle $(\bar{x}=1.4 \mu \mathrm{m}, \mathrm{n}=20)$, numerous, filamentous, septate, hyaline, branched at the base, swollen at the apices, strongly to very strongly capitates, terminal cell $2.5-4 \mu \mathrm{m}$ width $(\bar{x}=3.5 \mu \mathrm{m}, \mathrm{n}=20)$. Asci $35-45 \times 5.5-6.5 \mu \mathrm{m}(\bar{x}=41.2 \times 6.1 \mu \mathrm{m}, \mathrm{n}=30) 8$ spored, cylindric clavate, 8-spored, 1-2-seriate, with a short to long-stalked bifurcate base. 
Ascospores 3-3.5 $\times 1.2-1.6 \mu \mathrm{m}(\bar{x}=3.2 \times 1.4 \mu \mathrm{m}, \mathrm{n}=40)$, reniform to cashew-shaped, smooth walled. Asexual morph: Undetermined.

Material examined - THAILAND, Mae Fah Luang University, Chiang Rai Province, on unidentified wood, 20 April 2015, A.H. Ekanayaka, HD035 (MFLU 16-1861).

Notes - We were not able to obtain molecular data for this collection. However, by considering the morphological similarity of our collection with O. leucostigma (Fries 1849), we named our collection as Orbilia leucostigma. Orbilia leucostigma is morphologically similar to $O$. xanthostigma, but $O$. xanthostigma differs by having larger ascospores $(6-8.5 \times 1-1.4 \mu \mathrm{m})$ with warts (Guo et al. 2014, Quijada et al. 2016).

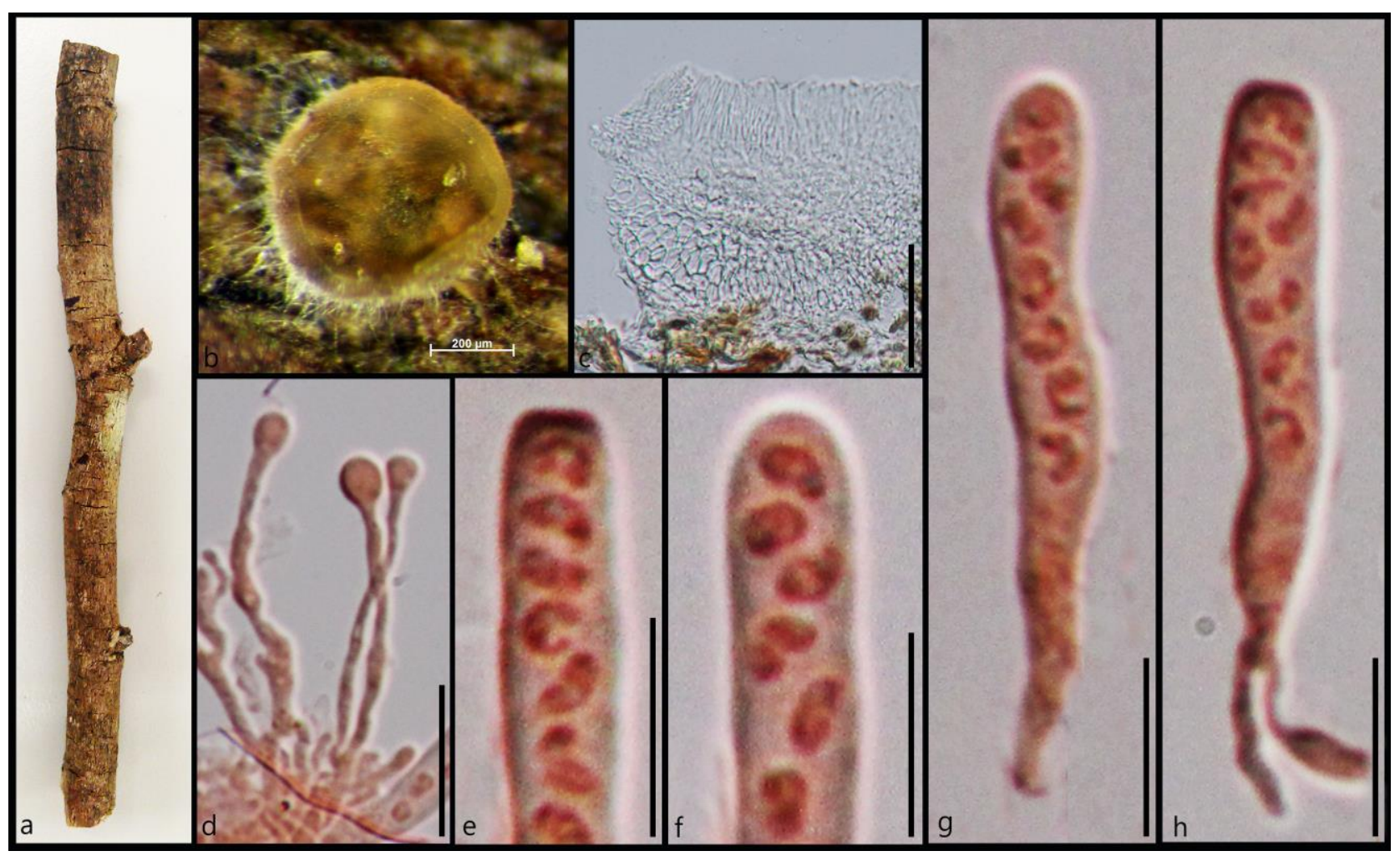

Figure 6 - Morphology of Orbilia leucostigma (MFLU 16-1861) a Substrate. b Fresh apothecia. c Excipular cells at upper flanks. d Paraphyses. e, f Asci. k-o Close up of ascus apex with ascospores. (d-h are mounted in congo red reagent) Scale bars: $b=200 \mu \mathrm{m}, \mathrm{c}=45 \mu \mathrm{m}, \mathrm{d}=35 \mu \mathrm{m}$, e and $\mathrm{f}=5$ $\mu \mathrm{m}, \mathrm{g}$ and $\mathrm{h}=10$.

\section{Acknowledgements}

This study was supported by the National Natural Science Foundation of China (No. 31360015) and the CAS/SAFEA International Partnership Program for Creative Research Teams, and the Knowledge Innovation Program of the Chinese Academy of Sciences (No. KSCX2-EW-Z9 KIB2016002). Anusha H. Ekanayaka is grateful to (Late) Mr. W. Ekanayaka, Mrs. C. Ekanayaka and Mr. A. Surasinghe for their valuable support and encouragement.

\section{References}

Baral HO, Weber E, Gams W, Hagedorn G et al. 2017 - Generic names in Orbiliaceae (Orbiliomycetes) and recommendations on which names should be protected or suppressed. Mycological Progress https://doi.org/10.1007/s11557-017-1300-6

Ekanayaka AH, Ariyawansa HA, Hyde KD, Jones EBG et al. 2017 - Discomycetes: The apothecial representatives of the phylum Ascomycota. Fungal Diversity 87, 237-298 
Eriksson OE, Baral HO, Currah RS, Hansen K et al. (eds.) 2003 - Outline of Ascomycota-2003. Myconet 9: 1-103.

Fries EM. 1849 - Summa Vegetabilium Scandinaviae 2, 259-572

Guo J, Li S, Yang L, Yang J et al. 2014 - New records and new distribution of known species in the family Orbiliaceae from China. African journal of Microbiology Research 8, 3178-3190

Hall T. 2004 - BioEdit. Ibis Therapeutics, Carlsbad, CA, 92008, USA. Available from: http://www.mbio.ncsu.edu/ BioEdit/bioedit.html (accessed December 2015).

Huelsenbeck JP, Ronquist F. 2001 - MRBAYES: Bayesian inference of phylogenetic trees. Bioinformatics 17, 754-755

Index Fungorum. 2017 - Available from: http://www.indexfungorum.org/Names/Names.asp. (accessed June 2016).

Jayasiri SC, Hyde KD, Ariyawansa HA, Bhat J et al. 2015 - The Faces of Fungi database: fungal names linked with morphology, phylogeny and human impacts. Fungal Diversity 74, 3-18.

Korf RP. 1973 - Discomycetes and Tuberales. In: Ainsworth GC, Sparrow FK, Sussman AS (eds) The fungi: an advanced treatise, vol IVA. Academic press, New York

Li Y, Hyde KD, Jeewon R, Cai L et al. 2006 - Phylogenetics and evolution of nematode-trapping fungi (Orbiliales) estimated from nuclear and protein coding genes. Mycologia 97, 10341046

Liu B, Liu XZ, Zhuang WY. 2005 - A new species of Hyalorbilia and its anamorph from China. Nova Hedwigia 81, 145-155

Miller MA, Pfeiffer W, Schwartz T. 2010 - Creating the CIPRES Science Gateway for inference of large phylogenetic trees. Gateway Computing Environments Workshop (GCE); 1-8.

Nannfeldt JA. 1932 - Studien über die Morphologic und Systematik der nichtlichenisierten non-operculaten Discomyceten. Nova Acta Regiae Societatis Scientiarum Upsaliensis 48, 1-368

Nylander JAA. 2004 - MrModeltest 2.0. Program distributed by the author. Evolutionary Biology Centre, Uppsala University.

Qiao M, Li JY, Baral HO, Zhang Y et al. 2015 - Orbilia yuanensis sp. nov. and its anamorph. Mycological Progress 14:1. doi: 10.1007/s11557-015-1022-6

Quijada L, Baral HO, Beltrán-Tejera E. 2012 - New species of Orbilia (Orbiliales) from arid ecosystems of the Canary Islands (Spain). Nova Hedwigia 96, 237-248.

Quijada L, Baral HO, Jaen-Molina R, Weiß M et al. 2014 - Phylogenetic and morphological circumscription of the Orbilia aurantiorubra group. Phytotaxa 175, 1-18

Quijada L, Baral HO, Beltran-Tejera E. 2015 - Diversity of Hyalorbilia (Orbiliales) in the Macaronesian Region. Nova Hedwigia 100, 1-14.

Quijada L, Baral HO, Beltrán-Tejera E. 2016 - A revision of the genus Orbilia in the Canary Islands. Phytotaxa 284, 231-262

Quijada L, Baral HO. 2017 - Orbilia beltraniae, a new succulenticolous species from the Canary Islands. MycoKeys 25, 1-12.

Rambaut A, Drummond AJ (2009) Tracer. MCMC Trace analysis tool version v1.5.0. http://tree.bio.ed.ac.uk/software/tracer/

Rannala B, Yang Z. 1996 - Probability distribution of molecular evolutionary trees: a new method of phylogenetic inference. Journal of Molecular Evolution 43, 304-311

Spooner BM. 1987 - Helotiales of Australasia: Geoglossaceae, Orbiliaceae, Scelrotiniaceae, Hyaloscyphaceae. Bibliotheca Mycologica 116, 1-711

Su HY, Zhang Y, Baral HO, Yang XY et al. 2011 - Four new species of Orbiliaceae from Yunnan, China. Mycological Progress 10, 373-381

Swofford DL. 2003 - PAUP*: phylogenetic analysis using parsimony (*and other methods), version 4. Sinauer Associates, Sunderland, Massachusetts

Vilgalys R, Hester M. 1990 - Rapid genetic identification and mapping of enzymatically amplified ribosomal DNA from several Cryptococcus species. Journal of Bacteriology 172, 4238-4246 
White TJ, Bruns T, Lee S, Taylor J. 1990 - Amplification and direct sequencing of fungal ribosomal RNA genes for phylogenetics. In: Innis MA, Gelfand DH, Sninsky JJ, White TJ(eds), PCR protocols a guide to methods and applications. Academic Press, Inc., New York, pp 315-322.

Yu ZF, Qiao M, Zhang Y, Baral HO, Zhang KQ. 2007 - Orbilia vermiformis sp. nov. and its anamorph. Mycotaxon 99, 271-278

Yu ZF, Qiao M, Zhang Y, Li Q, Zhang KQ. 2011 - Pseudotripoconidium, a new anamorph genus connected to Orbilia. Mycologia 103, 164-173

Zhang Y, Yu ZF, Baral HO, Mo MH, Zhang KQ. 2009 - New species and new Chinese records of the genus Orbilia (Orbiliaceae, Ascomycota) from Yunnan, China. Fungal Diversity 36, 141153

Zhaxybayeva O, Gogarten JP. 2002 - Bootstrap, Bayesian probability and maximum likelihood mapping: exploring new tools for comparative genome analyses. BMC Genomics 3, 4-22 